\title{
APPLICATIONS OF DIGITAL CORRELATION METHOD TO STRUCTURE INSPECTION
}

\author{
G. C. Jin \\ Dept. of Engineering Mechanics, \\ Tsinghua University, Beijing, China \\ Phone:8610-62772914, \\ Fax:8610-62781824, \\ E-mail:jingc@mail.tsinghua.edu.cn
}

\author{
J.D. Chen \\ Dept. of Engineering Mechanics, \\ Tsinghua University, Beijing, $n$ China \\ Phone:8610-62772914, \\ Fax:8610-62781824, \\ E-mail: @mails.tsinghua.edu.cn
}

\author{
L.B. Meng \\ Dept. of Engineering Mechanics, Tsinghua University, Beijing China \\ Phone:8610-62785586, Fax:8610-62781824 \\ E-mail:mlb00@mails.tsinghua.edu.cn
}

\begin{abstract}
In the structure inspection, non-destructive inspection technique (NDT) becomes an important tool for safe operation of structure especially in nuclear structure. A non-contact optical deformation measurement technique named Digital Correlation Method (DCM) has been developed and applied to structure inspection.

In this paper, the principle of an improved DCM is introduced. The finite element smoothing technique with two-dimensional GCV algorithm is developed in this paper that provides the high precision strain measurement.

Three structure inspection examples are listed in this paper. In the first example, the interface shear stress distribution of reinforced concrete piles bedded on rock was inspected. The result of strain measurement show that stress distribution of reinforced concrete-rock interface appears a stress concentration on upside and fast decrease with bedded depth and it agree well with the in situ measurement and FEM analysis.

The second example is a 3D strain distribution of composite vessel structure. In this structure, the normal direction (Z) stress is an important inspection task. For the measurement of $\varepsilon_{\mathrm{z}}$, A 3D correlation technique is developed, and the strain measurement $\varepsilon_{\mathrm{z}}$ shows the structure is on the elastic stage. It also provides the safety evaluation for the composite vessel. The final example is the stress inspection of a hookup that connects two steel bridge structures, a large rigid-body displacement will be appears on the loading. Two compensation methods are developed that can remove the large rigid body movement. The measuring result shows the huge stress concentration on the hookup and it agree with the design data.

The elemental advantages of DCM in the nuclear engineering are presented and the prospect of these advanced technique on structure engineering is discussed.
\end{abstract}

Keywords: Structure inspection, DCM, 3D strain measurement

\section{INTRODUCTION}

In structure inspection, non-destructive inspection technique (NDT) becomes important tool for safe operation of structure especially in nuclear structure. A non-contact optical deformation measurement technique named Digital Correlation Method (DCM) has been developed in the end of last century. ${ }^{[1]}$ The principle of DCM is to correlate two speckle images on the structure surface and to obtain deformation data 
of structure between two speckle images captured before and after deformed. The random speckle patterns that formed by artificial or by natural means are captured by a CCD camera then processed by computer. Software that consists of the correlation or iteration algorithm, an image search feature and sub-pixel processing functions, is used for calculating the deformation information. Many improvements that provide the higher precision and higher processing speed of DCM are completed ${ }^{[2-4]}$. Usually the displacement measuring precision of DCM can reach to 0.01 pixels or $1 \mu \mathrm{m}$ for $10 \mathrm{~cm}$ object and the processing time can less than several seconds. For the strain measurement, an important problem is how to obtain strain distribution from the displacement distribution. It is obviously if the displacement field has a little deviation or noise factor, the strain will appear large error. One of important improvement that uses a finite element smoothing technique with two-dimensional GCV algorithm is developed in this paper, thus an optimized displacement field is extracted.

The main advantages of DCM are non-contact, full field and easy to set in situ; the non-contact means it can be applied in various environment conditions even in high temperature, radiation environment; full field means the measurement is not just a point and it can easy to find the strain concentration position. DCM is easy to set and used in suit, it only need CCD camera to record the random speckle patterns that can be natural or artificial, if ridge body displacement exist, DCM still work well. DCM can be used not in Lab but in outdoor. It is obviously the DCM becomes a perfect tool in structure inspection or reliability research of structure.

In this paper, three measurement examples are provided that includes the reinforced concrete-rock structure (interface stress), it provide a criterion for designing the reinforced concrete-rock interface. For the vessel stress measurement, a 3D strain measurement technique is developed, two cameras are used for recording the images of same sample with different direction and the space relationship will be applied to resolve the 3D displacement. A calibration work is necessary to obtain the space relationship precisely. The last stress measurement of metal hookup of steel bridge shows the huge stress concentration but also on the design data range. The solutions of classical structure inspection proved the DCM was the one of best tools for structure inspection especially in nuclear engineering.

\section{THE PRINCIPLE OF DCM}

In general, a spatial-domain cross-correlation function is used to describe the correspondences, on the basis of the spatial-domain cross-correlation function. Ma and Jin $^{[5]}$ compared different correlation functions, concluded the properties of them, and then a new cross-correlation function with high sharpness was presented and listed as Eq.1

$$
C_{2}=C^{2}=\frac{\left\{\sum \sum[(f-\bar{f}) \cdot(g-\bar{g})]\right\}^{2}}{\sum \sum(f-\bar{f})^{2} \cdot \sum \sum(g-\bar{g})^{2}}
$$

Another approach of describing the correspondences is called iterative spatial-gradient-based algorithm. This algorithm calculate the first-order spatial derivatives of the images instead of calculating the correlation coefficient, it can reduces the computation complexity and saves time due to huge correlation calculation. But usually the strain data are not perfect, and even are bad when the noise is at a high level. In this paper, a smoothing method is applied to the raw displacement field obtained by iteration, and then the smoothed data field is differentiated to provide the estimated 'noiseless' displacement gradients.

In Ref. ${ }^{[6]}$, finite-element method was used to smooth full-field experimental data such as displacement or strain field. The basic concept is to find a desired smoothing function $d$ by minimizing the following function $\mathrm{R}(\mathrm{d})$

$$
R(d)=\frac{1}{2} \sum_{i=1}^{J}\left(d_{i}-\widehat{d}_{i}\right)^{2}+\frac{\lambda}{2} \iint_{A}\left(\left(\frac{\partial^{2} d}{\partial x^{2}}\right)^{2}+\left(\frac{\partial^{2} d}{\partial y^{2}}\right)^{2}+2\left(\frac{\partial^{2} d}{\partial x \partial y}\right)^{2}\right) d A
$$

where

$\widehat{d}_{i}$ : displacement component to be imposed at points i ( $u$ or $v$ )

$d_{i}$ : smoothed solution value for $\hat{d}_{i}$ 
$J$ : number of total points

A : minimum region included all points

$\lambda$ : smoothing parameter

Region A is divided into Q elements in the usual way of finite-element method, and the smoothed solution value $\mathrm{d}$ in the jth element can be expressed as

$$
d=[N]_{j}\{a\}_{j},
$$

$[N]_{j}$ : shape functions of the jth element

$\{a\}_{j}$ : column vector of the jth element degrees of freedom

After substituting equation (3) into equation (2), the following equation is obtained.

$$
\begin{gathered}
R=\frac{1}{2} \sum_{j=1}^{Q}\left\{\left\{a^{e}\right\}_{j}{ }^{T}\left[K_{1}^{e}\right]_{j}\left\{a^{e}\right\}_{j}-2\left\{a^{e}\right\}_{j}{ }^{T}\{P\}_{j}+\right. \\
\left.\lambda\left\{a^{e}\right\}_{j}{ }^{T}\left[K_{2}^{e}\right]\left\{a^{e}\right\}_{j}\right\}+ \text { Const }
\end{gathered}
$$

where

$$
\begin{aligned}
{\left[K_{1}^{e}\right]_{j}=} & \sum_{i=1}^{S_{j}}\left[N_{i}\right]_{j}{ }^{T}\left[N_{i}\right]_{j}, \quad\{P\}_{j}=\sum_{i=1}^{S_{j}}[N]_{j}^{T} \widehat{d}_{i j}, \\
{\left[K_{2}^{e}\right]_{j}=} & \iint_{D_{j}}\left\{\left(\frac{\partial^{2}[N]_{j}}{\partial x^{2}}\right)^{T}\left(\frac{\partial^{2}[N]_{j}}{\partial x^{2}}\right)+2\left(\frac{\partial^{2}[N]_{j}}{\partial x \partial y}\right)^{T}\left(\frac{\partial^{2}[N]_{j}}{\partial x \partial y}\right)+\right. \\
& \left.\left(\frac{\partial^{2}[N]_{j}}{\partial y^{2}}\right)^{T}\left(\frac{\partial^{2}[N]_{j}}{\partial y^{2}}\right)\right\} d A
\end{aligned}
$$

where

$S_{j}$ : the number of total points in jth element

$\left[N_{i}\right]_{j}$ : the value of $[N]_{j}$ at ith point of jth element

$\widehat{d}_{i j}$ : displacement component to be imposed at ith point of jth element

$\left[K_{1}^{e}\right]_{j},\left[K_{2}^{e}\right]_{j}$ are called element stiffness matrix, and $\{P\}_{j}$ is called element load vector. The global-equation assembly process is conducted in the usual way, and the result is as follows:

$$
R=\frac{1}{2}\{a\}^{T}\left[K_{1}\right]\{a\}-\{a\}^{T}\{P\}+\lambda\{a\}^{T}\left[K_{2}\right]\{a\}+\text { Const }
$$

Minimization of equation (13) by variation of the coefficients $\{a\}$ results in the normal equations:

$[K]\{a\}=\{P\}, \quad[K]=\left[K_{1}\right]+\lambda\left[K_{2}\right]$.

If the smoothing parameter $\lambda$ is known, nodal parameter $\{a\}$ can be resulted from equation (6). In Ref. ${ }^{[7]}$, the effect of $\lambda$ was discussed. If the $\lambda$ is zero, then no smoothing occurs and the formulation (6) produces a least-square fit of the experimental data. If the $\lambda$ is very large, then maximum smoothing occurs and formulation (6) produces a fit of plane to the experimental data. For obtaining a good estimation of standard deviation for displacement field, the one-dimensional GCV algorithm was introduced ${ }^{[6]}$. However, $\lambda$ is only an empirical value, but not determined definitely in Ref. ${ }^{[6-7] .}$ In this paper, two-dimensional GCV algorithm is introduced to estimate the smoothing parameter $\lambda$. GCV algorithm involves choosing $\lambda \geq 0$ to minimize the function 


$$
\begin{aligned}
& G C V(\lambda)=\frac{n\|[I-A(\lambda) P]\|}{\{\operatorname{tr}[I-A(\lambda)]\}^{2}}, \\
& A(\lambda)=\left[K_{1}\right]\left(\left[K_{1}\right]^{T}\left[K_{1}\right]+\lambda\left[K_{2}\right]\right)^{-1}\left[K_{1}\right]^{T} .
\end{aligned}
$$

In Ref ${ }^{[8]}$, for obtaining the globally optimal value of $\lambda$ for two-dimensional or higher-dimensional problems, a complete description of an algorithm based on interval analysis is given. Then $\{a\}$ is obtained, and thus the smoothed data field may be determined from equation (3), and it is differentiated to provide the estimated 'noiseless' displacement gradients.

\section{TESTING EXAMPLES}

\subsection{Concrete-rock interface stress measurement}

In the base construction of bridge engineering, reinforced concrete bed in rock call wall-type pier is a best way that may save more in engineering, it is obviously the stress distribution of interface between reinforced concrete and rock is very important for the load bearing. DCM is the best tool for this interface stress measurement application.

For the new wall-type pier of great highway bridge design, a concrete-rock model experiment was proposed. Four 1:30 scaled models made of granite and concrete was adopted. DSCM was applied in this experiment and the artificial speckle pattern was painted on the surface, the speckle pattern is shown on Fig.1. A 100 T testing machine was used for loading the model. The shear stress distribution of the interface was obtained and shown in Fig.2.

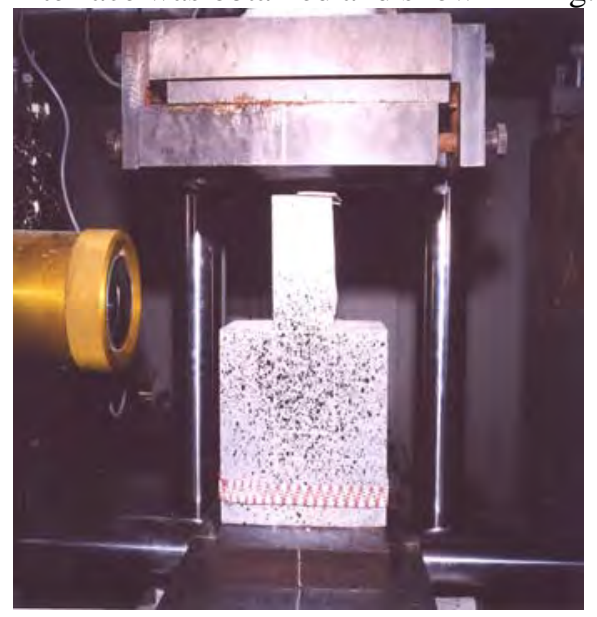

Fig.1 Speckle pattern of pier model

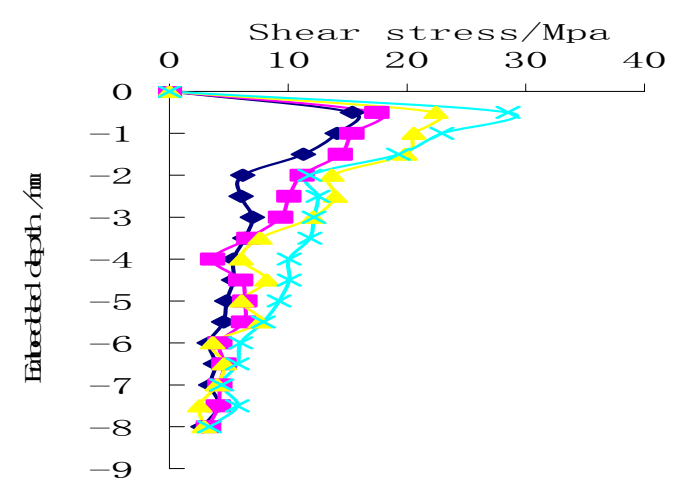

Fig.2. The shear stress distribution of four models

The results of experimental study show the maximum stress is concentrated on the upper side of the interface and reduced very quickly; the interface will bear the large parts of the load of the bridge. It agrees well with the FEM calculation, thus the base of bridge design is full of safety. After the end of engineering a real test was completed and the measurements of shear stress distribution is same as the model testing. From the results of experimental and FEM, a simplification formula is introduced for other bridge design.

\section{2 composite pressure vessel}

For the simple structure, usually a 2D DSCM is enough to deduct the plane stress field, but for the complex structure, 3D DSCM is necessary. The principle of 3D DSCM is the dual camera arrangement that formed a space geometry. If the relation between the object coordinate system and camera coordinates system obtained, the 3D displacement field of measured object can be determined. There are four coordinate systems and 11 parameters must be determined. It includes 3 parameters of rotate matrix, 3 parameters of translation matrix, focus distance $f$, diametric distortion coefficient $k$, factor 
$\lambda$, and the coordinate origin of CCD. In our research, a two-step calibration technique is adopted based on the diametric restriction and the orthogonality of rotation matrix. The calibration system is consisted of two CCD cameras set arbitrarily and a standard square target placed on a 3D translation platform. A standard displacement of the square target is recorded by two cameras and it repeats several times. From these calibration tests, the space geometry parameter can be deducted and the test error of 3D displacement is about $3 \%$.

For the composite structure, the strain testing of a vessel made of carbon fiber is completed. The vessel is consisted by muti-layer carbon fibers that has different direction $0^{\circ}$ (horizontal), $45^{\circ},-45^{\circ}$. The vessel and CCD camera are shown in Fig.3.

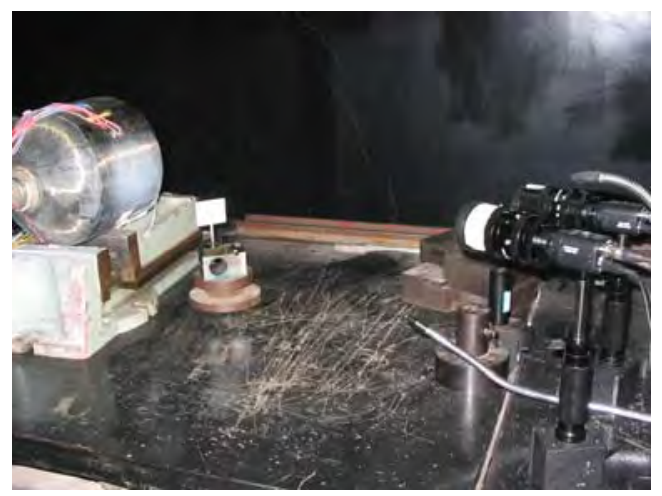

Fig.3 the experimental set-up

An oil pump is used for the loading apparatus and the load is from 0-3MPa. A serious speckle patterns are recorded by two cameras simultaneously and a correlation program is used to deduct the 3D displacement data. The displacement $\mathrm{Z}$ distribution is shown in Fig.4. A curve, which displays the stress -strain is shown in Fig.5 and it was still on the elastic stage.

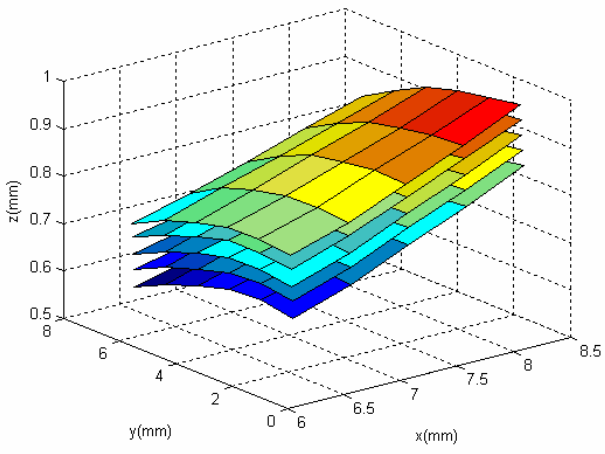

Fig 4.The displacement distribution.

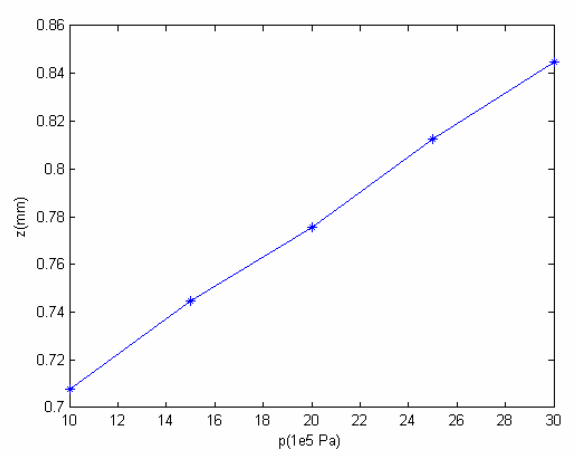

Fig.5. The $\sigma-\varepsilon$ curve of the composite vessel

\subsection{Steel structure inspection}

The large structure inspection is an important problem in Engineering. Reactor, large bridge is a typical and popular large structure, these structures always construct by concrete and steel. In this paper we presented a real steel bridge inspection for proving the possibility and reliability of this new tool.

A movable steel bridge is used as a sample and a hookup is used to connect the two parts of bridge, the stress concentration exists on the hookup. To inspection it on the loading condition is important for the safety and reliability of the movable steel bridge. Due to the large rigid-body displacement, it always reduces the correlation and moves the undeformed speckle pattern outside the view field. An in-site camera fixation and removable camera set are developed and it overcomes the trouble of large rigid-body displacement than 
obtains the perfect results. The artificial speckle pattern is formed on the measured hookup surface. Two speckle patterns are recorded before and after loading, a heavy vehicle is driven on the bridge as the loading. The bridge and DSCM set-up is shown in Fig.7a and the measuring area is shown in Fig.7b. The measuring result shows a great stress concentration on the bending place of hookup (Fig.8). The maximum strain is reach to $2500 \mu \varepsilon$ and it agree well with the calculate results.

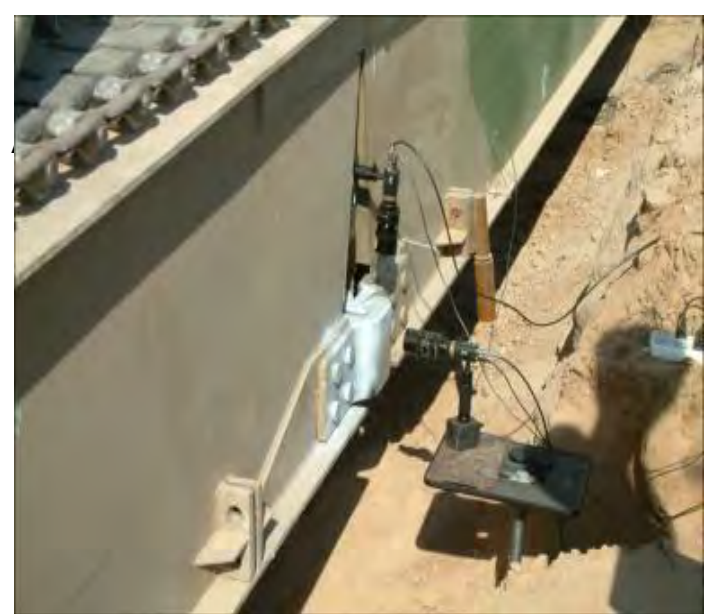

Fig. 7a. The photo of bridge and DSC set-up.

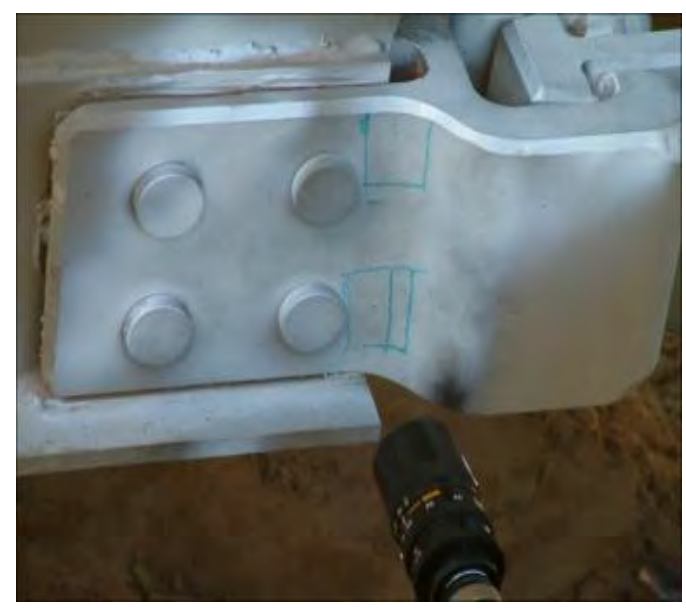

Fig.7b. The hookup and measurement area

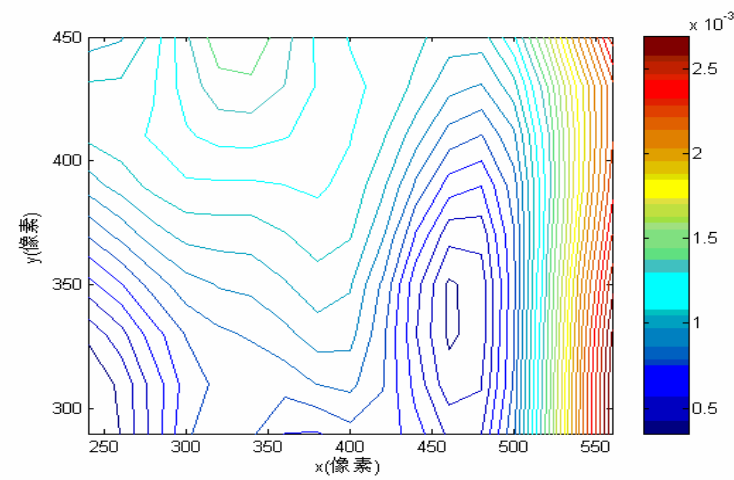

Fig.8. The strain distribution of the hookup

\section{CONCLUSION}

From three examples, it is clearly the DSC is a new tool for the structure inspection tools. These examples show the method is very suitable for the large structure inspection also in reaction engineering.

\section{REFERENCE}

[1] Peters WH and Ranson WF, "Digital Imagine techniques in experimental stress analysis”, Optical Engineering, 1982, 21:427-432

[2] Chu T C, Ranson W F, Sutton M A and Peter W H, "Applications of Digital Image-Correlation Technique to Experimental Mechanics,” Exp.Mech. 1985, 25, 232-244

[3] Rui Jiabai, Jin Guanchang, Xu Bingye, “A New Digital Speckle Correlation Method And Its Application”, ACTA. MECHANICA. SINICA, 1994, 26(5), 599-607

[4] Peng Zhou, Kenneth E.Goodson, "Subpixel displacement and deformation gradient measurement using digital image/speckle correlation(DISC)”, Opt.Eng. 2001, 40(8), 1613-1620

[5] Ma Shaopeng, Jin Guanchang, "New correlation coefficients designed for digital speckle correlation method (DSCM)”, Proceedings of SPIE - The International Society for Optical Engineering, 2003, 5058, 25-33 
[6] M.A. Sutton, J.L.Turner, H.A.Bruck and T.A. Chae, "Full-field Representation of Discretely Sampled Surface Deformation for Displacement and Strain Analysis”, Exp.Mech, 1991, 31(2), 168-177

[7] Segalman, D.J., Woyak, D.B. and Rowlands, R.E., "Smooth spline-like Finite-element Differentiation of Full-field Experimental Data over Arbitrary Geometry,” EXPERIMENTAL MECHANICS, 19(12), 429-437(1979)

[8] J.T.KENT and M.MOHAMMADZADEH, "Global optimization of the generalized cross-validation criterion", Statistics and Computing, 2000, 10, 231-236 